\title{
EFFETS DE CONDITIONS MICROCLIMATIQUES DIFFÉRENTES SUR LA MORPHOLOGIE ET LA STRUCTURE ANATOMIQUE DES AIGUILLES DE QUELQUES RÉSINEUX
}

\author{
G. AUSSENAC \\ Station de Sylviculture et Production, \\ Centre national de Recherches forestières, I.N.R.A. \\ Champenoux 54370 Einville
}

\begin{abstract}
RÉSUMÉ
L'étude entreprise porte sur 5 résineux : Abies alba (Ecouves II), Abies alba (Canigou), Abies nordmanniana (Gerbéviller), Picea abies (La Joux), Pseudotsuga menziesii (Farges II). Les plants ont été installés dans 7 traitements différents :

- sous une coupe d'abri (densité 1300 tiges/ha, hauteur moyenne $13 \mathrm{~m}$ ),

- dans 5 situations (Nord, Sud, Est, Ouest, Centre) d'une clairière circulaire ( $26 \mathrm{~m}$ de diamètre, hauteur moyenne du peuplement environnant $13 \mathrm{~m}$ ),

- en plein découvert. l'air.

Ces traitements diffèrent essentiellement par le microclimat lumineux et le pouvoir évaporant de

Les études ont consisté en mensurations, coupes d'aiguilles, comptages et mesures des stomates. Il y a une relation étroite entre la forme des aiguilles et le niveau d'énergie reçu. D'une façon générale, les aiguilles de la coupe d'abri sont plates alors qu'elles sont beaucoup plus épaisses dans le plein découvert. Le rapport $\frac{\text { largeur }}{\text { épaisseur }}$ décroît depuis les éclairements relatifs faibles (coupe d'abri) jusqu'aux éclairements les plus forts. Il en est de même pour le rapport des diagonales de la section des aiguilles de l'Épicéa. Le rapport $\frac{\text { longueur }}{\text { largeur }}$ varie avec l'éclairement relatif et passe par un maximum dans la clairière. Il en est de même pour la longueur des aiguilles.

Chez le Douglas et Abies alba (Ecouves II) le nombre de stomates augmente avec l'éclairement et est ainsi le plus élevé dans le plein découvert. Le Douglas a une numération stomatique plus élevée et des stomates plus petits que les Sapins. Ces caractéristiques sont peut-être l'indice d'une meilleure régulation de la transpiration.

Les résultats de cette étude permettent de comprendre la crise de découvert chez les plants ayant poussé à l'ombre et qui sont brusquement éclairés par enlèvement du couvert.

Quand le niveau d'énergie reçu par les arbres est très bas (14\%) les aiguilles des résineux de la coupe d'abri ont une morphologie et une anatomie très différentes de celle des aiguilles de plein découvert. Lorsque le niveau d'énergie augmente, les aiguilles s'avèrent « incapables » d'utiliser l'énergie dispensée. Au contraire, les plants de la clairière présentent des aiguilles assez voisines de celles du plein découvert et sont capables de bénéficier d'un éclairement important. Ce type de traitement permet un passage sans problème de l'abri temporaire au plein découvert. Ainsi, lorsque la coupe rase n'est pas supportable, le gestionnaire de forêt doit s'orienter vers une coupe d'abri aussi claire que possible ou, mieux encore, vers des coupes par bandes.
\end{abstract}




\section{I. - INTRODUCTION}

Les jeunes arbres se développent et croissent dans des conditions microclimatiques variées qui dépendent essentiellement de la structure et de l'importance du couvert végétal préexistant. Selon les cas : terrain découvert, clairière, abri des cimes, leur croissance est plus ou moins rapide et leur appareil foliaire «s'adapterait » aux conditions de milieu. Ainsi, on parle de feuilles d'ombre et de feuilles de lumière (Perrin, 1963). Les feuilles d'ombre ont un fonctionnement différent des feuilles de lumière. La photosynthèse se manifeste avec des éclairements plus faibles que chez les feuilles de lumière. Mais, elle est vite ralentie quand l'intensité lumineuse croît et elle atteint son maximum pour des éclairements relativement faibles (BOYSEN JENSEN, 1932; RousSel, 1970). Selon les conditions microclimatiques, une même essence ou provenance peut donc présenter des feuilles de l'une ou l'autre catégorie ou même des deux dans le cas des houppiers d'arbres adultes. Ainsi, l'étude des influences du microclimat sur l'appareil foliaire peut, en nous montrant un certain nombre d'évolutions, nous mettre sur la voie d'une meilleure compréhension du fonctionnement de l'arbre.

Les caractères morphologiques et anatomiques des feuilles des arbres, et particulièrement des résineux, ont été étudiés par un certain nombre d'auteurs (DAGUILlon, 1890, etc...), mais peu d'entre eux (Mer, 1883, Dufour, 1887) se sont attachés à l'étude de l'influence des facteurs écologiques sur les feuilles. Ainsi, la morphologie et l'anatomie comparée des aiguilles de conifères croissant en conditions microclimatiques différentes sont mal connues. Le présent rapport fait état des études entreprises à Nancy sur les aiguilles de quelques résineux.

\section{II. - MATÉRIELS ET MÉTHODES}

L'étude entreprise porte sur 5 résineux dont les caractéristiques principales sont données par le tableau suivant (tableau 1).

TABLEAU 1

Caractéristiques des espèces et provenances étudiées

Caracteristics of studied species and provenances

\begin{tabular}{|c|c|c|}
\hline Espèces & Provenance & Age \\
\hline Abies alba Mill. & $\begin{array}{l}\text { Ecouves II. Lat. } 48^{\circ} 31^{\prime}-\text { Long. } 0^{\circ} 04^{\prime} \mathrm{E} \\
\text { Orne (France). Altitude } 300 \mathrm{~m}\end{array}$ & 9 ans \\
\hline Abies alba Mill. & $\begin{array}{l}\text { Canigou. Lat. } 42^{\circ} 33^{\prime}-\text { Long. } 2^{\circ} 27^{\prime} \mathrm{E} \\
\text { Pyrénées-Orientales (France). Altitude } 1800 \mathrm{~m}\end{array}$ & 9 ans \\
\hline $\begin{array}{l}\text { Abies nordmanniana } \\
\text { Spach. }\end{array}$ & $\begin{array}{l}\text { Gerbéviller. Lat. } 48^{\circ} 30^{\prime}-\text { Long. } 6^{\circ} 30^{\prime} \text { E } \\
\text { Meurthe-et-Moselle (France). Altitude } 300 \mathrm{~m}\end{array}$ & 9 ans \\
\hline Picea abies L. & $\begin{array}{l}\text { La Joux. Lat. } 46^{0} 51^{\prime} \text { L Long. } 6^{0} 03^{\prime} \mathrm{E} \\
\text { Jura (France). Altitude } 800 \mathrm{~m}\end{array}$ & 9 ans \\
\hline $\begin{array}{l}\text { Pseudotsuga menziesii } \\
\text { Mirb. }\end{array}$ & $\begin{array}{l}\text { Farges II. Lat. } 45^{\circ} 32^{\prime}-\text { Long. } 2^{\circ} 11^{\prime} \mathrm{E} \\
\text { Corrèze (France). Altitude } 700 \mathrm{~m}\end{array}$ & 9 ans \\
\hline
\end{tabular}

Les plants ont été installés dans sept traitements différents :

- sous une coupe d'abri constituée par un taillis de charmes, hêtres, chênes, tilleuls, d'une densité de 1300 tiges/ha et d'une hauteur moyenne de $13 \mathrm{~m}$, 
- dans une clairière circulaire de $26 \mathrm{~m}$ de diamètre située au sein d'un peuplement forestier ayant des caractéristiques identiques au précédent. Dans cette clairière, 5 situations particulières sont distinguées : Nord, Sud, Est, Centre, Ouest,

- en plein découvert dans une coupe rase.

Les traitements considérés diffèrent essentiellement par le microclimat lumineux et le pouvoir évaporant de l'air :

\begin{tabular}{l|c|c}
\hline \multicolumn{1}{c|}{ Traitement } & $\begin{array}{c}\text { Éclairement } \\
\text { en \% du plein découvert (1) }\end{array}$ & $\begin{array}{c}\text { Pouvoir évaporant de l'air } \\
\text { en \% du plein découvert (2) }\end{array}$ \\
\hline Coupe d'abri (C.A.) & 9 & 58 \\
Clairière Nord (C.N.) & 35 & 62 \\
Clairière Sud (C.S.) & 14 & \\
Clairière Est (C.E.) & 22 & 100 \\
Clairière Centre (C.C.) & 45 & \\
Clairière Ouest (C.O.) & 22 & \\
Plein découvert (P.D.) & 100 & \\
\hline
\end{tabular}

(1) Mesuré au luxmètre en été par temps couvert pour la coupe d'abri et calculé à partir des chiffres publiés par Roussel pour la clairière.

(2) Moyenne mai-octobre 1968, mesuré au Piche.

Le dispositif expérimental est situé en forêt domaniale d'Amance à $15 \mathrm{~km}$ à l'est de Nancy. Les conditions topographiques et climatologiques générales sont identiques : replat à $250 \mathrm{~m}$ d'altitude sur sol brun lessivé à pseudogley sur marnes liasiques.

Dans chaque traitement, 20 aiguilles (âgées d'un an) ont été prélevées sur 5 arbres. Ces aiguilles étaient situées sur le deuxième verticille à partir du sommet de l'arbre. Les études effectuées ont consisté en mensurations, coupes d'aiguilles, comptages et mesures des stomates.

Les coupes ont été faites au milieu des aiguilles; le colorant utilisé est le carmino vert de Mirande.

Les comptages et les mesures des stomates sont faits à partir de photographies réalisées avec un épimicroscope ZEISS. Les photographies sont ensuite projetées et examinées sur écran.

\section{III. - RÉSULTATS}

\section{1. - Effet du microclimat sur la morphologie des aiguilles}

\section{Sur les dimensions et la forme des aiguilles}

Les dimensions des aiguilles des 5 espèces ou provenances ont été étudiées dans les 7 situations écologiques retenues. Trois paramètres de base sont considérés : la longueur (L), la largeur (1) et l'épaisseur (figure 1). Dans le cas de l'épicea, ce sont les diagonales (D et d) de la section de l'aiguille qui ont été mesurées (voir figure 1). Les figures 2 et 3 donnent les différentes valeurs obtenues et les classements respectifs des stations étudiées.

La longueur des aiguilles est la plus courte dans les traitements de plein découvert (P.D.) et de coupe d'abri (C.A.) pour les deux provenances d'Abies alba. (Les traits verticaux des figures joignent les stations qui ne sont pas statistiquement différentes au seuil $\mathbf{P}=0,05)$. Les aiguilles des différentes stations de la clairière sont d'une façon générale plus longues. Pour l'ensemble, les valeurs s'étagent de 25,3 mm (C.E.) à 18,3 mm (P.D.), pour Abies alba Ecouves II, et de 25,0 mm (C.N.) à 15,8 mm (P.D.) pour Abies alba Canigou. 
Chez Abies nordmanniana, les résultats sont un peu différents. Cependant ici aussi, les aiguilles des plants poussant en plein découvert sont les plus courtes. Les longueurs s'étagent entre $28,1 \mathrm{~mm}$ (C.C.) et 20,3 mm (P.D.). Ici encore, les différences entre les aiguilles de la coupe d'abri et celles du plein découvert ne sont pas significatives au seuil de probabilité choisi $(\mathrm{P}=0,05)$.
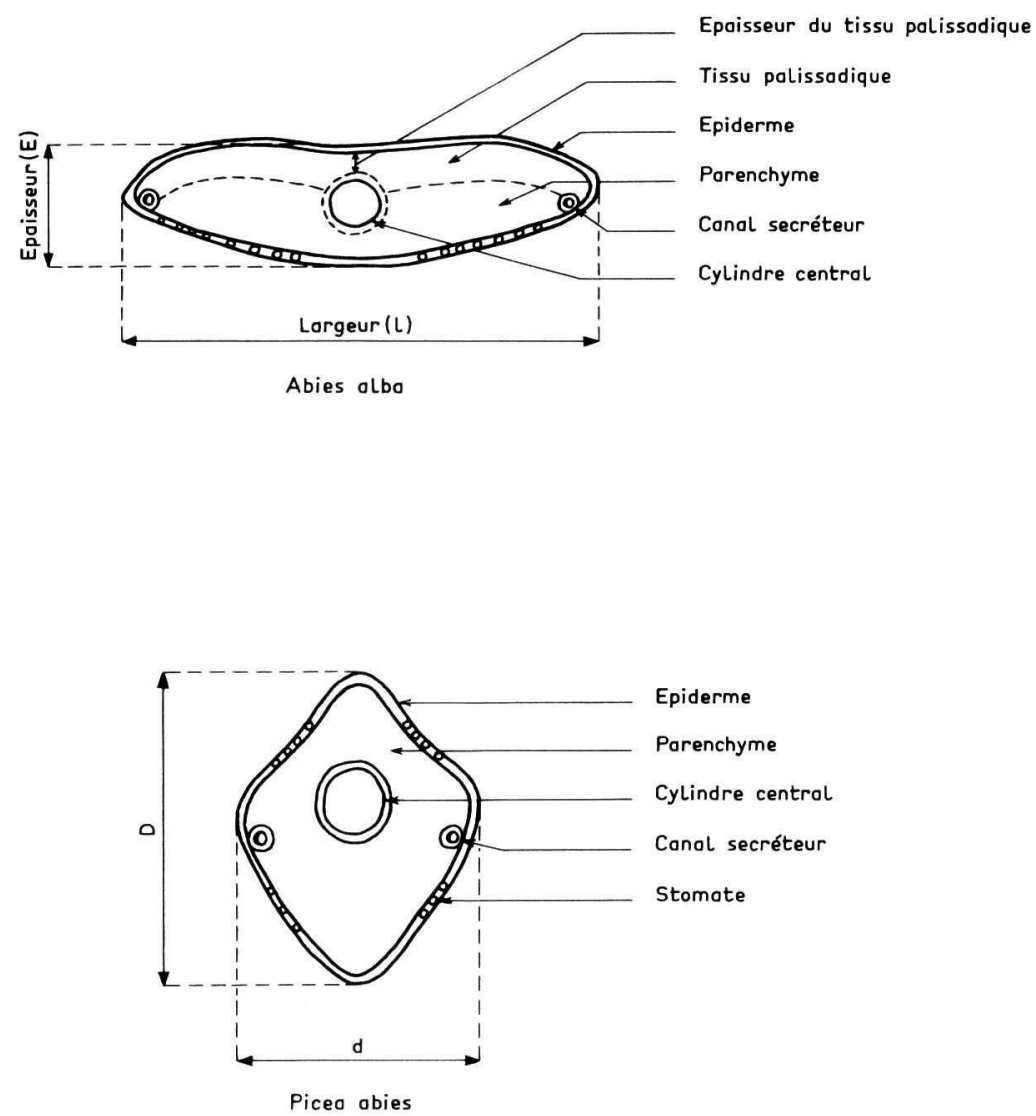

FIG. 1. - Coupe schématique d'une aiguille de Sapin pectiné et d'une aiguille d'Épicéa avec les paramètres étudiés

Section of a fir needle and section of a Spruce needle, with the studied parameters

Au contraire, dans le cas de Pseudotsuga menziesii, ce sont les aiguilles des arbres poussant dans la coupe d'abri (C.A.) qui sont les plus courtes. La différence avec les autres traitements est statistiquement significative.

Les aiguilles du plein découvert sont moins longues que celles des différentes stations de la clairière, mais les différences ne sont pas significatives. Pour l'ensemble, les longueurs vont de 33,0 mm (C.N.) à 22,5 mm (C.A.).

En ce qui concerne la largeur (figures 2 et 3 ) les aiguilles des deux provenances d'Abies 


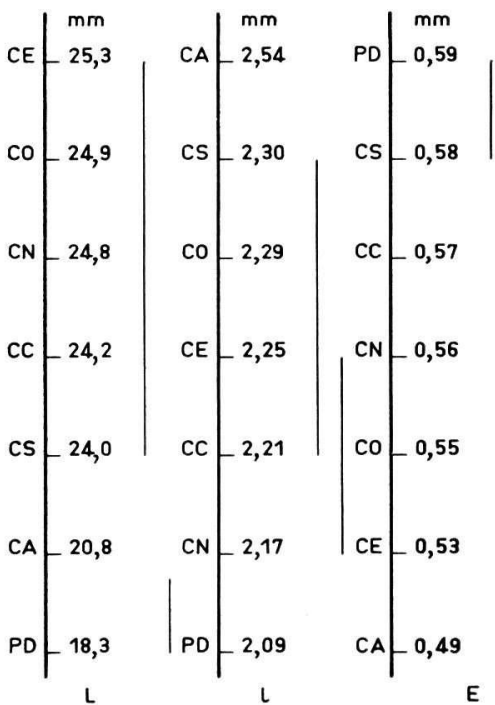

Ecouves

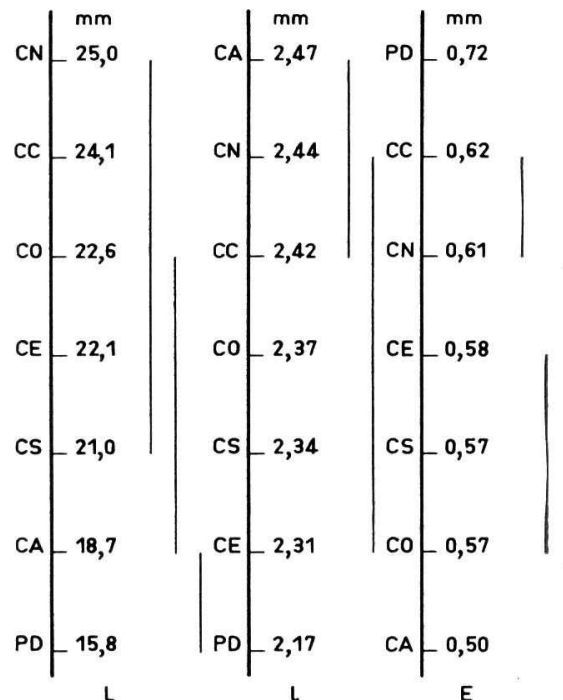

Canigou

\section{Abies alba}

FIG. 2. - Dimensions ( $\mathrm{mm}$ ) (L : longueur, 1 : largeur, E : épaisseur) des aiguilles.

Les traits réunissent des traitements qui ne sont pas statistiquemen différents

Dimensions of needles $(\mathrm{mm})(\mathrm{L}:$ length, $1:$ width, E: thickness) The traces join statistically identical treatments

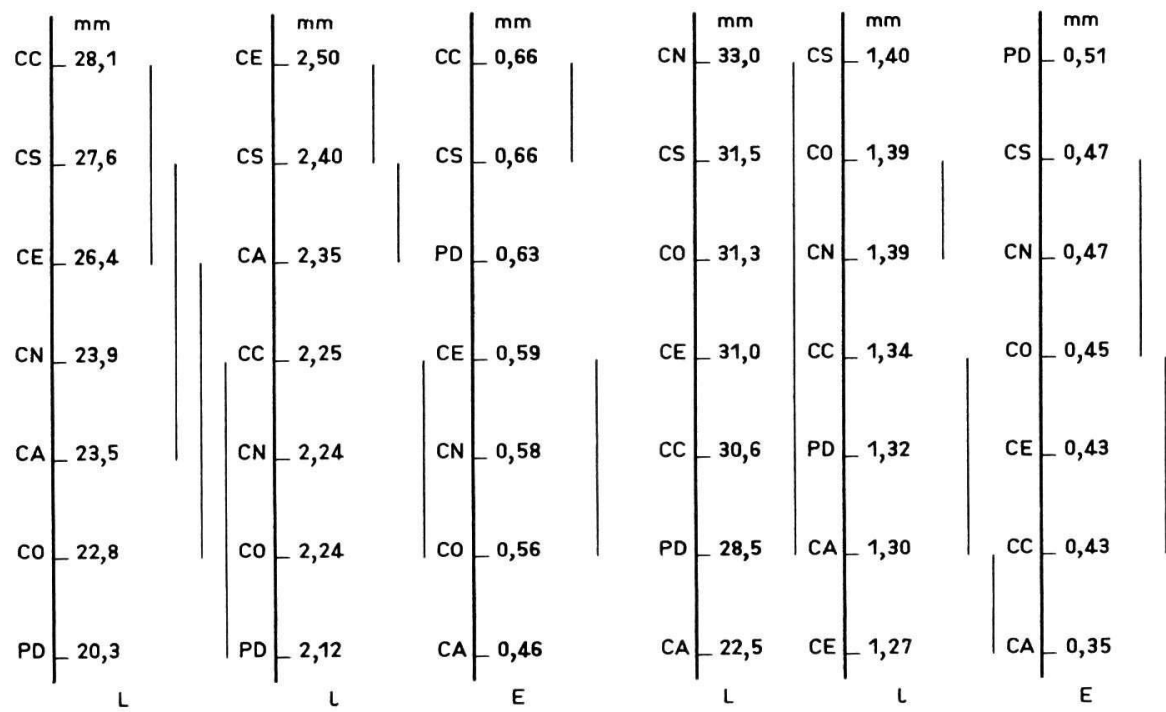

FIG. 3. - Dimensions ( $\mathrm{mm}$ ) (L : longueur, I : largeur, E : épaisseur) des aiguilles. Les traits réunissent des traitements qui ne sont pas statistiquement différents

Dimensions of needles ( $\mathrm{mm}$ ) ( $\mathrm{L}:$ length, 1 : width, $\mathrm{E}:$ thickness) The traces join statistically identical treatments 
alba sont les plus larges dans la coupe d'abri (2,54 mm Abies alba Ecouves II, 2,47 mm Abies alba Canigou) et les plus étroites dans le plein découvert $(2,09 \mathrm{~mm}$ Abies alba Ecouves II, 2,17 mm Abies alba Canigou). Les aiguilles des plants des différentes stations de la clairière ont une position intermédiaire. Chez Abies nordmanniana, on note une différence : les aiguilles les plus larges se rencontrent non plus dans la coupe d'abri, mais dans l'est de la clairière (C.E.) $(2,5 \mathrm{~mm})$. Les aiguilles les plus étroites sont celles du plein découvert (2,12 $\mathrm{mm})$. Dans le cas du Pseudotsuga menziesii, les choses sont très différentes puisque ce ne sont ni les aiguilles de coupe d'abri qui sont les plus larges, ni les aiguilles du plein découvert qui sont les plus étroites. Ce sont les différentes stations de la clairière qui couvrent l'ensemble de la variation, des valeurs les plus élevées (C.S. : 1,40 mm) aux plus petites (C.E. : 1,27 mm). Ainsi, le Douglas apparaît ici très différent du Sapin pectiné et du Sapin de Nordmann.

Le rapport $\mathbf{L} / 1$ varie selon les cas (figure 6). Il est d'une façon générale plus faible dans la coupe d'abri et le plein découvert que dans les différentes stations de la clairière qui ont donc des aiguilles proportionnellement plus longues.

Les aiguilles sont les plus épaisses dans le plein découvert et les moins épaisses dans la coupe d'abri pour les deux provenances d'Abies alba et pour Pseudotsuga menziesii (figures 2 et 3). Pour le Sapin de Nordmann, les aiguilles de la coupe d'abri sont aussi les moins épaisses, mais ce sont les aiguilles du centre de la clairière qui ont la plus grande épaisseur.

L'épicéa présente (figure 1) des aiguilles dont la section est sensiblement un losange. Les mesures de la grande diagonale (D) et de la petite diagonale (d) sont données par la figure 4 . C'est dans la coupe d'abri (C.A.) que l'on trouve simultanément les dimensions les plus faibles (D : 0,98 mm, d : 0,50 mm). Les valeurs les plus élevées se rencontrent dans la partie ouest de la clairière (C.O.) pour la grande diagonale $(\mathrm{D}: 1,26 \mathrm{~mm})$ et dans le plein découvert

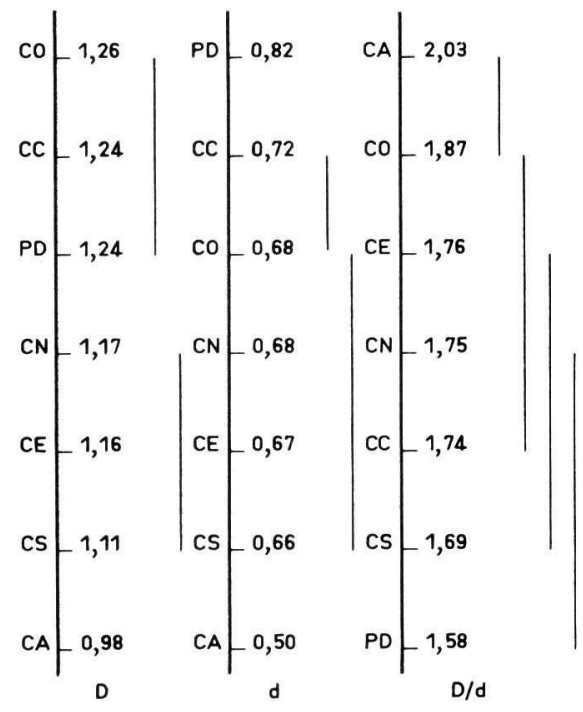

Piceo abies

FIG. 4. - Dimensions ( $\mathrm{mm}$ ) des diagonales (D et d) des aiguilles d'Épicéa.

Les traits réunissent les traitements qui ne sont pas statistiquement différents

Size of the diagonals ( $D$ and d) of Spruce Needles

The traces join statistically identical treatments 
(P.D.) pour la petite diagonale $(0,82 \mathrm{~mm})$. Le rapport $\mathrm{D} / \mathrm{d}$ qui donne une bonne idée de la forme de la section de l'aiguille est le plus faible $(1,58)$ dans le plein découvert et le plus grand dans la coupe d'abri $(2,03)$.

Chez les autres résineux étudiés, le rapport largeur/épaisseur 1/E permet de préciser la forme de la section des aiguilles. Il est le plus élevé dans la coupe d'abri et le plus faible dans

FIG. 5. - Coupes typiques dans les aiguilles

Typical Sections in the needles

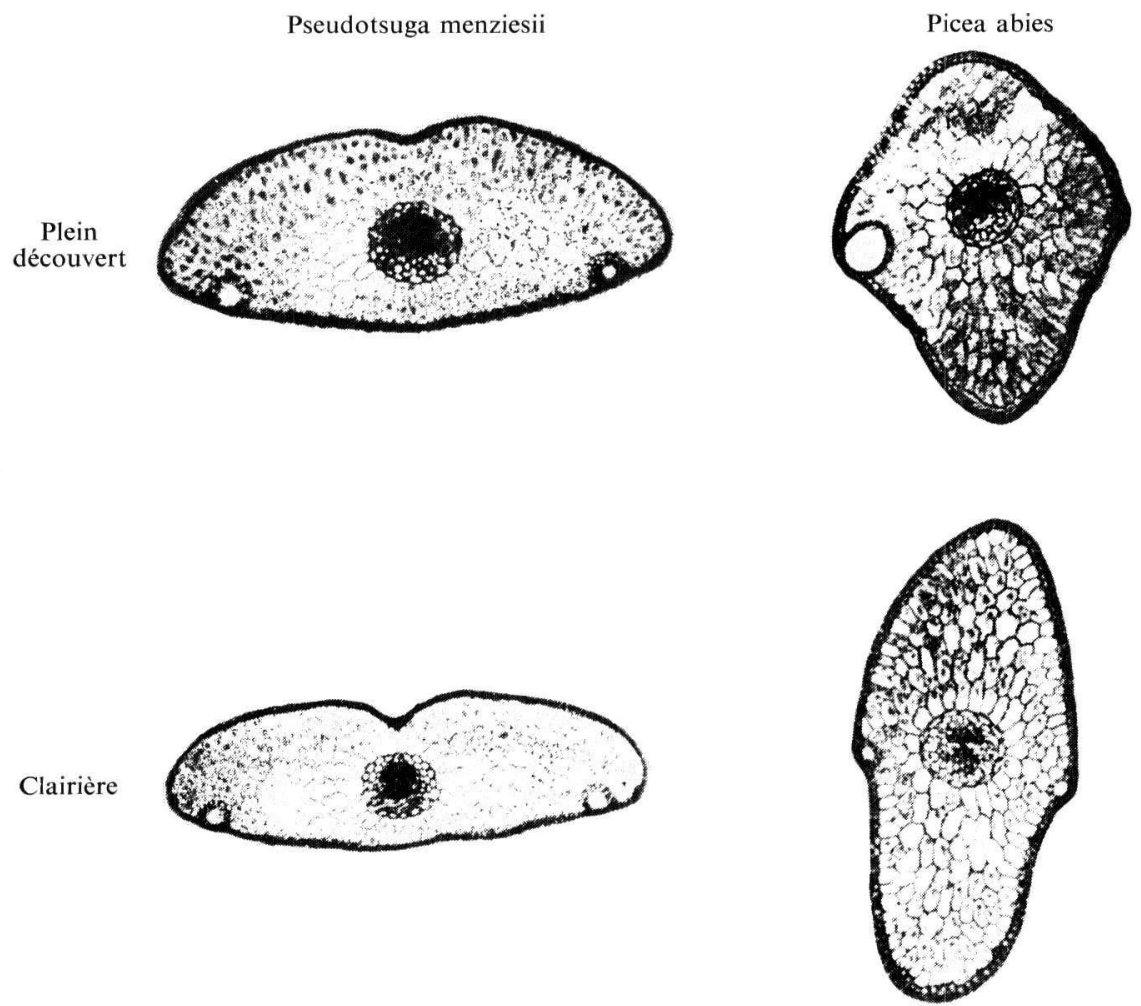

Coupe
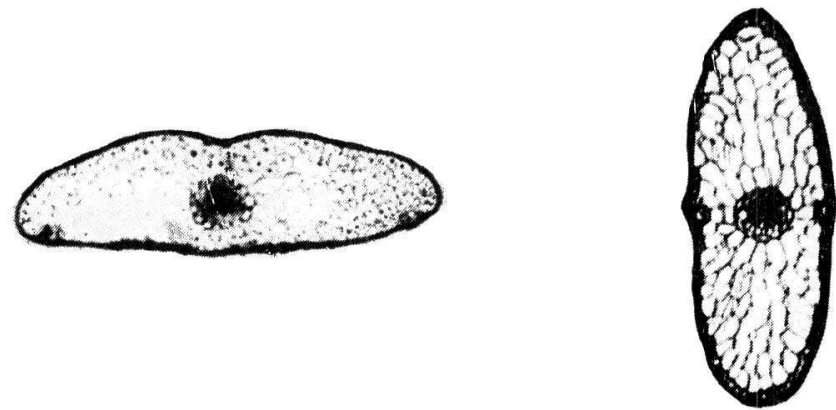
FIG. 5. (suite) - Coupes typiques dans les aiguilles

Typical sections in the needles

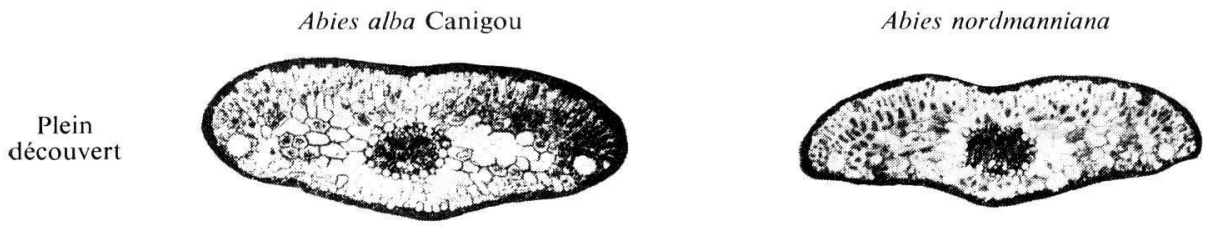

Abies nordmanniana

Plein découvert

Clairière
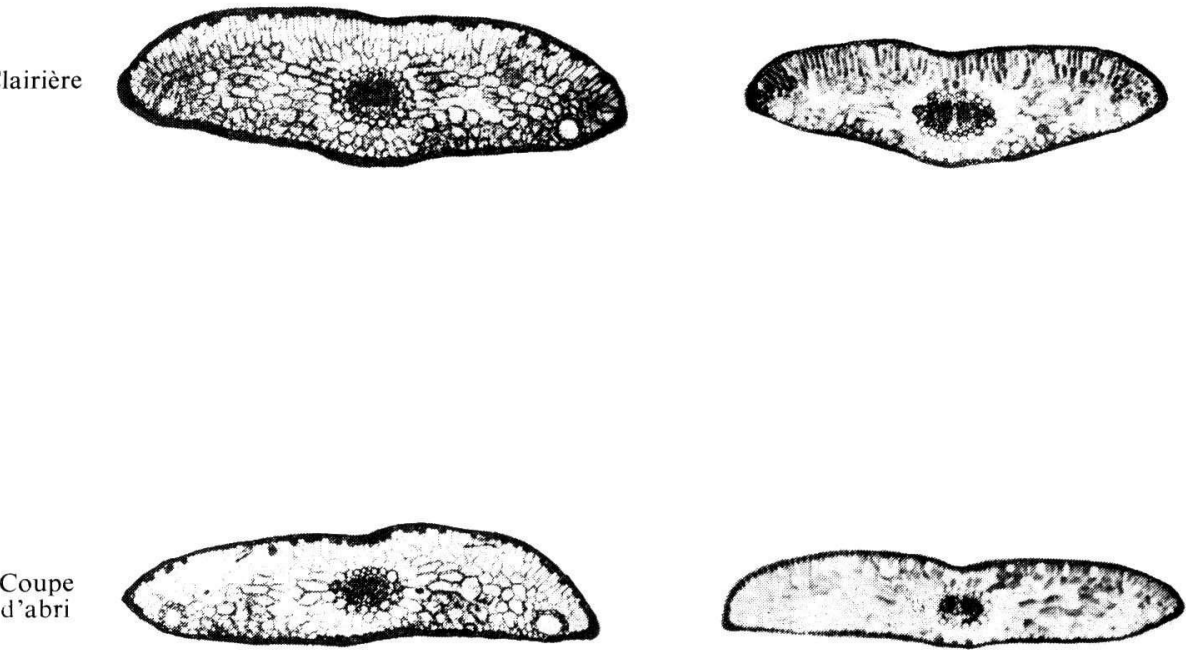

le plein découvert pour les 4 espèces ou provenances (figure 7). Les aiguilles sont aplaties dans la coupe d'abri et « globuleuses 》 dans le plein découvert ainsi que le montrent les photographies de la figure 5. Les aiguilles des différentes stations de la clairière ont une forme intermédiaire.

Le rapport L/1 (figure 6) varie avec l'éclairement relatif et passe par un maximum dans la clairière. Il en est de même pour la longueur (L) des aiguilles.

Le rapport $1 / \mathrm{E}$ décroît depuis les éclairements relatifs faibles (coupe d'abri) jusqu'aux éclairements les plus forts. Il en est de même de D/d pour l'Épicéa (figure 7).

Il y a donc une relation étroite entre la forme des aiguilles et le niveau d'énergie reçu. Quand l'éclairement est faible les aiguilles sont aplaties.

\section{Sur le nombre et la dimension des stomates}

Le tableau 2 donne les valeurs obtenues pour les différents paramètres étudiés (nombre de stomates $/ \mathrm{mm}^{2}$ de surfaces des bandes stomatiques, nombre de stomates $/ \mathrm{mm}^{2}$ de surface 


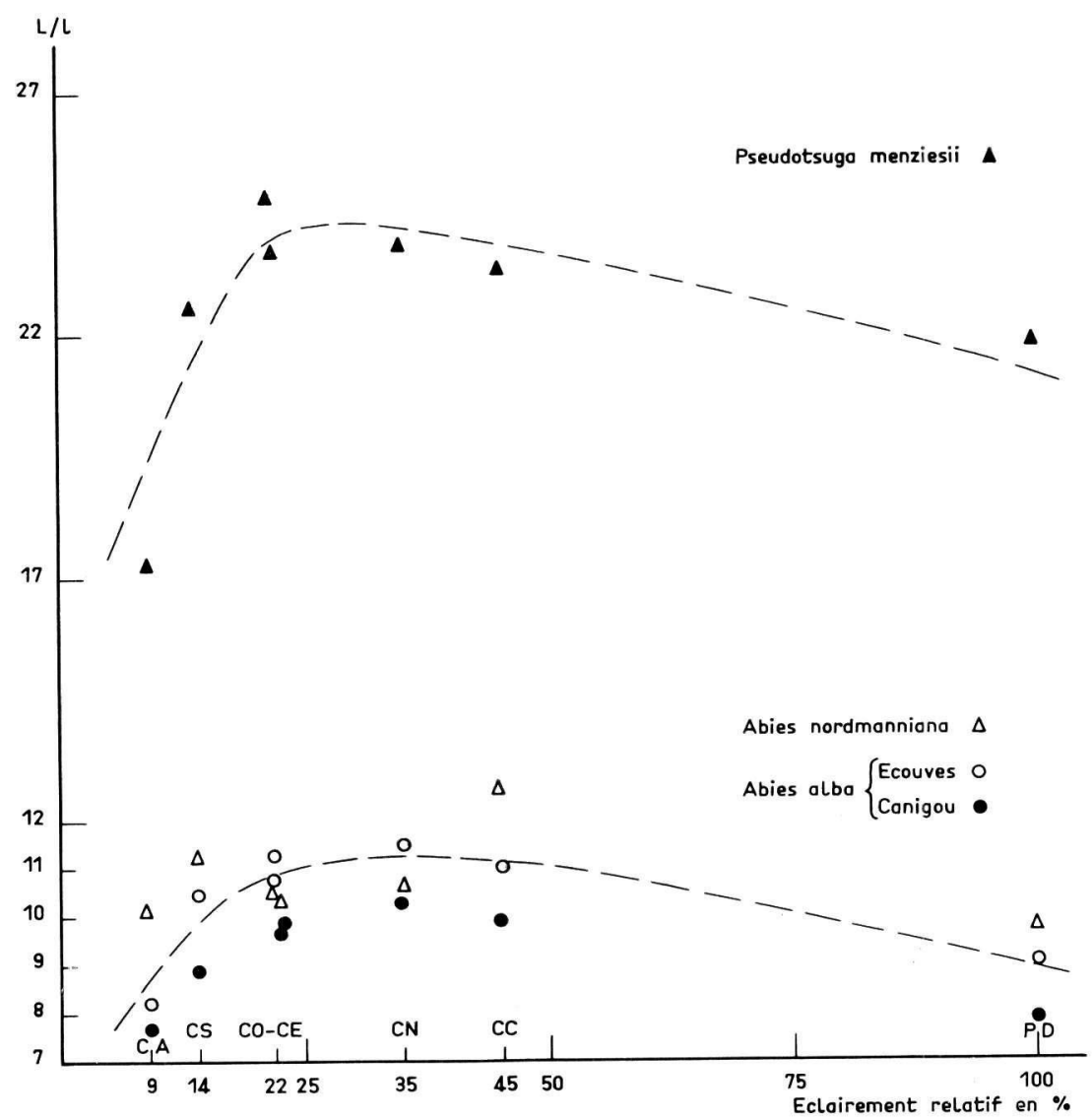

FIG. 6. - Variations du rapport longueur (L) / largeur (1) des aiguilles en fonction de l'éclairement relatif Variations of ratio: $\mathrm{L} / 1$ in function of relative illumination

foliaire, largeur des bandes stomatiques, nombre de files de stomates). Le nombre de stomates par $\mathrm{mm}^{2}$ de surface foliaire varie de 60 à $80 \mathrm{chez}$ Abies alba Ecouves II, de 62 à $78 \mathrm{chez}$ Abies alba Canigou, de 63 à 86 chez Abies nordmanniana et de 110 à 142 chez Pseudotsuga menziesii. Ainsi que l'indique le tableau 2, les effets traitements ne sont pas toujours significatifs.

Les dimensions des stomates ont aussi été évaluées. L'analyse de variance montre que l'effet traitement n'est significatif pour aucune des espèces étudiées (voir tableau 3 ). Chez les trois sapins étudiés ces dimensions sont voisines. Le rapport $\frac{\text { longueur des stomates }}{\text { largeur des stomates }}$ montre qu'en moyenne les stomates d'Abies alba Ecouves et Abies nordmanniana sont plus arrondis que ceux d'Abies alba Canigou.

Les dimensions des stomates du Douglas sont nettement inférieures à celles des Sapins. 

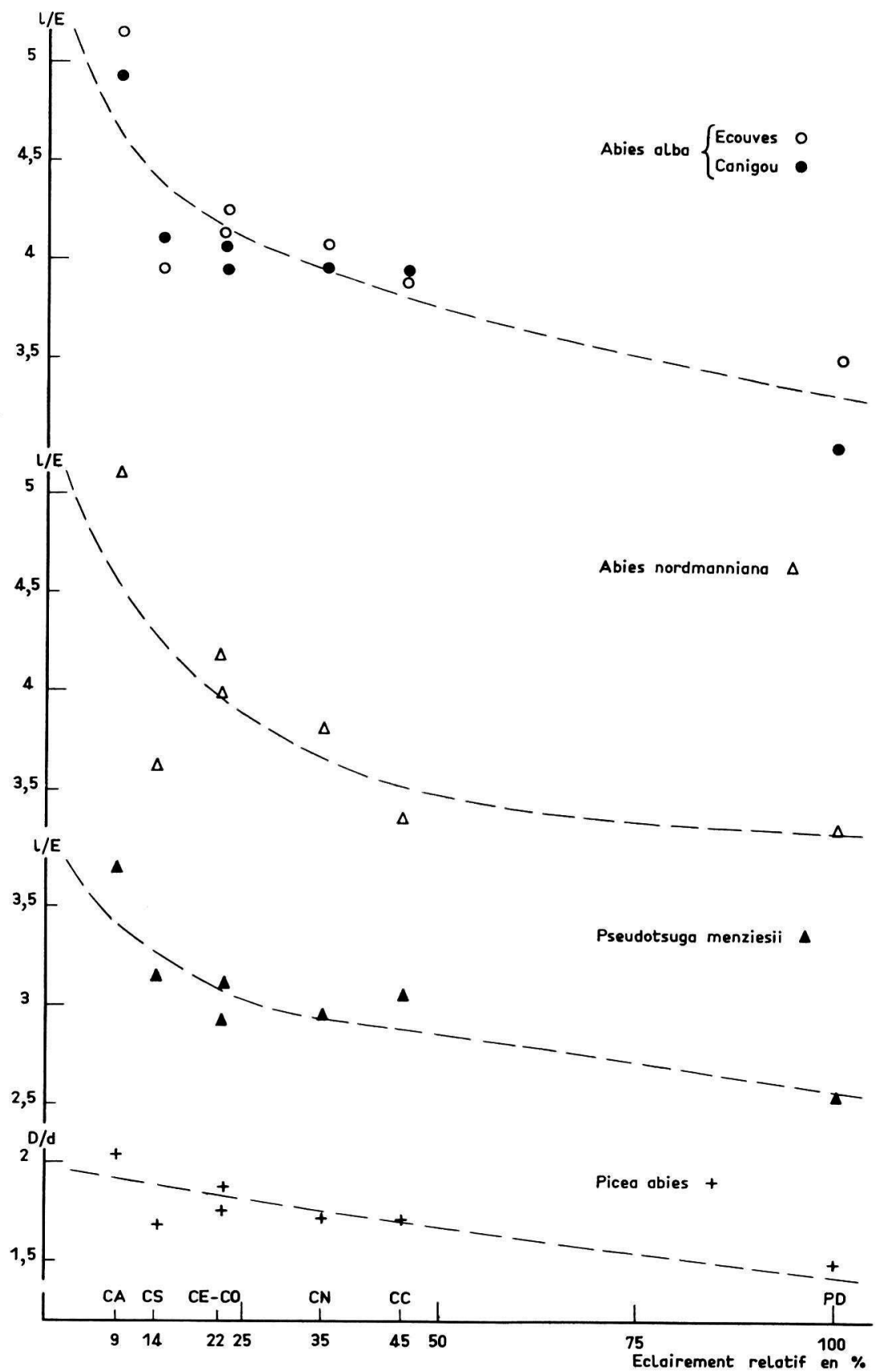

FIG. 7. - Variation du rapport largeur (1) / épaisseur (E) et du rapport $\mathrm{D}$ (grande diagonale) / d (petites diagonales) en fonction de l'éclairement relatif

Variations of ratios $1 / \mathrm{E}$ and $\mathrm{D} / \mathrm{d}$ in function of relative illumination 


\section{TABLEAU 2}

Effet du traitement sur le nombre de stomates, la largeur des bandes stomatiques et le nombre de files de stomates. Test de signification * significatif à 0,05

Effect of treatment on the number stomatas, the width of stomatic strips and the number of stomatas rows

\begin{tabular}{|c|c|c|c|c|c|c|c|c|c|c|}
\hline Espèce & Paramètre & C.A. & C.N. & C.S. & C.E. & C.C. & C.O. & P.D. & $\begin{array}{l}\text { D.L. } \\
(1)\end{array}$ & $\mathrm{F}$ \\
\hline \multirow{3}{*}{$\begin{array}{l}\text { Abies alba } \\
\text { Ecouves II }\end{array}$} & $\begin{array}{l}\text { Nbre de stomates } / \mathrm{mm}^{2} \text { bande } \\
\text { stomatique }\end{array}$ & 173 & 178 & 160 & 176 & 178 & 177 & 210 & $\begin{array}{r}8 \\
28\end{array}$ & $4,3 * *$ \\
\hline & $\begin{array}{l}\text { Nbre de stomates } / \mathrm{mm}^{2} \text { sur- } \\
\text { face foliaire }\end{array}$ & 65 & 72 & 60 & 68 & 75 & 65 & 80 & $\begin{array}{r}6 \\
28\end{array}$ & $3,8^{*}$ \\
\hline & $\begin{array}{l}\text { Largeur bande stomatique } \\
\quad \text { en } \mathrm{mm}\end{array}$ & 0,427 & 0,447 & 0,435 & 0,423 & 0,470 & 0,432 & 0,380 & $\begin{array}{r}6 \\
28\end{array}$ & 1,3 N.S. \\
\hline \multirow{3}{*}{$\begin{array}{l}\text { Abies alba } \\
\text { Canigou }\end{array}$} & $\begin{array}{l}\text { Nbre de stomates } / \mathrm{mm}^{2} \text { bande } \\
\text { stomatique }\end{array}$ & 175 & 160 & 171 & 167 & 159 & 162 & 173 & $\begin{array}{r}6 \\
20\end{array}$ & 1,1 N.S. \\
\hline & $\begin{array}{l}\text { Nbre de stomates } / \mathrm{mm}^{2} \text { sur- } \\
\text { face foliaire }\end{array}$ & 62 & 70 & 66 & 70 & 67 & 68 & 65 & $\begin{array}{r}6 \\
20\end{array}$ & 0,5 N.S. \\
\hline & $\begin{array}{l}\text { Largeur bande stomatique } \\
\text { en } \mathrm{mm}\end{array}$ & 0,428 & 0,498 & 0,437 & 0,470 & 0,502 & 0,490 & 0,394 & $\begin{array}{r}6 \\
20\end{array}$ & $4,5^{* *}$ \\
\hline \multirow{3}{*}{$\begin{array}{l}\text { Abies } \\
\text { nordmanniana }\end{array}$} & $\begin{array}{l}\text { Nbre de stomates } / \mathrm{mm}^{2} \text { bande } \\
\text { stomatique }\end{array}$ & 170 & 184 & 172 & 181 & 190 & 185 & 192 & $\begin{array}{r}6 \\
26\end{array}$ & 0,9 N.S. \\
\hline & $\begin{array}{l}\text { Nbre de stomates } / \mathrm{mm}^{2} \text { sur- } \\
\text { face foliaire }\end{array}$ & 63 & 85 & 83 & 82 & 83 & 86 & 79 & $\begin{array}{r}6 \\
26\end{array}$ & $3,7^{*}$ \\
\hline & $\begin{array}{l}\text { Largeur bande stomatique } \\
\text { en } \mathrm{mm}\end{array}$ & 0,421 & 0,471 & 0,555 & 0,524 & 0,486 & 0,498 & 0,424 & $\begin{array}{r}6 \\
26\end{array}$ & $6,8^{* *}$ \\
\hline \multirow{4}{*}{$\begin{array}{l}\text { Pseudostuga } \\
\text { menziesii }\end{array}$} & $\begin{array}{l}\text { Nbre de stomates } / \mathrm{mm}^{2} \text { bande } \\
\text { stomatique }\end{array}$ & 279 & 263 & 258 & 258 & 286 & 278 & 297 & $\begin{array}{r}6 \\
26\end{array}$ & 1,08 N.S. \\
\hline & $\begin{array}{l}\text { Nbre de stomates } / \mathrm{mm}^{2} \text { sur- } \\
\text { face foliaire }\end{array}$ & 110 & 129 & 124 & 123 & 130 & 131 & 142 & $\begin{array}{r}6 \\
26\end{array}$ & 1,6 N.S. \\
\hline & $\begin{array}{l}\text { Largeur bande stomatique } \\
\quad \text { en } \mathrm{mm}\end{array}$ & 0,236 & 0,320 & 0,337 & 0,293 & 0,290 & 0,315 & 0,313 & $\begin{array}{r}6 \\
26\end{array}$ & $3,6 *$ \\
\hline & Nbre de files de stomates $\left({ }^{2}\right)$ & 5 & 6 & 6 & 6 & 6 & 6 & 6 & $\begin{array}{r}6 \\
26\end{array}$ & $2,9 *$ \\
\hline
\end{tabular}

(1) D.L. nombre de degrés de liberté (2) par 1/2 aiguille. N. S. : non significatif. 
TABLEAU 3

Effet du traitement sur les dimensions en microns $\left(\mu^{\prime}\right)$ des stomates Effect of treatment upon dimensions (u) of stomatas

\begin{tabular}{|c|c|c|c|c|c|c|c|c|}
\hline Espèce & Paramètre & C.A. & C.N. & C.S. & C.E. & C.C. & C.O. & P.D. \\
\hline \multirow{2}{*}{$\begin{array}{l}\text { Abies alba } \\
\text { Ecouves II }\end{array}$} & Longueur (L) & 75,7 & 76,9 & 77,3 & 78,1 & 73,9 & 78,1 & 71,2 \\
\hline & Largeur (1) & 50,1 & 49,5 & 51,9 & 50,4 & 52,4 & 51,0 & 49,5 \\
\hline \multirow{2}{*}{$\begin{array}{l}\text { Abies alba } \\
\text { Canigou }\end{array}$} & Longueur & 72,5 & 77,6 & 77,7 & 80,0 & 82,4 & 81,1 & 73,1 \\
\hline & Largeur & 56,5 & 56,7 & 55,2 & 58,7 & 58,5 & 61,0 & 52,6 \\
\hline \multirow{2}{*}{$\begin{array}{l}\text { Abies } \\
\text { nordmanniana }\end{array}$} & Longueur & 73,0 & 78,9 & 75,2 & 79,4 & 74,4 & 78,3 & 75,8 \\
\hline & Largeur & 49,2 & 57,9 & 54,6 & 54,8 & 54,9 & 56,1 & 52,9 \\
\hline \multirow{2}{*}{$\begin{array}{l}\text { Pseudotsuga } \\
\text { menziesii }\end{array}$} & Longueur & 49,2 & 47,7 & 48,4 & 51,0 & 47,1 & 47,7 & 49,7 \\
\hline & Largeur & 30,9 & 32,1 & 32,5 & 33,4 & 34,8 & 31,6 & 37,3 \\
\hline
\end{tabular}

\section{2. - Effet sur la structure des aiguilles}

L'étude a porté sur l'ensemble des tissus, mais n'ont été retenus ici que les caractères intéressants au regard de leurs réactions aux variations microclimatiques (figure 1). L'épaisseur du tissu palissadique $\left(\mathrm{t}_{\mathrm{p}}\right)$ a été mesurée au niveau du cylindre central (tableau 4). L'analyse statistique a montré que chez toutes les espèces étudiées ici, l'effet traitement était significatif au seuil de $\mathrm{P}=0,05$. Chez Abies alba (Ecouves II et Canigou) et Pseudotsuga menziesii l'épaisseur du tissu palissadique est la plus importante dans le plein découvert et la plus faible dans la coupe d'abri. Dans l'ensemble, ce tissu apparaît plus épais chez le Sapin pectiné (Canigou) que chez Abies alba (Ecouves II). Chez Abies nordmanniana, le tissu palissadique est plus épais dans le centre de la clairière (C.C.). Le rapport épaisseur aiguille (E) /épaisseur tissu palissadique $\left(t_{p}\right)$ (tableau 5 ) est d'une façon générale le plus fort dans la coupe d'abri, ce qui signifie que pour des aiguilles d'épaisseur égale, le tissu palissadique est moins épais. Le cylindre central a un diamètre variable (tableau 4), mais qui est toujours le plus faible dans la coupe d'abri.

Le diamètre des canaux résinifères est, d'une façon générale, plus petit dans la coupe d'abri (tableau 4). D'autres caractères anatomiques ont été analysés, mais il n'était pas possible d'effectuer à partir d'eux une étude biométrique correcte compte tenu de l'importance de l'échantillon nécessaire.

\section{IV. - DISCUSSION ET CONCLUSIONS}

Dans l'ensemble, il apparaît qu'il y a un effet significatif du traitement sylvicole sur la forme et la structure des aiguilles. Cependant il existe une grande variabilité au niveau des arbres ainsi que l'ont montré les analyses de variance. Il est possible de différencier trois grands types d'aiguilles, caractéristiques du plein découvert, de la coupe d'abri et de la clairière. A l'intérieur de cette dernière, il est difficile de caractériser chaque situation. Pour 


\section{TABLEAU 4}

Épaisseur moyenne du tissu palissadique $\left(\mathrm{t}_{\mathrm{p}}\right)$, rapport Épaisseur de l'aiguille (E) / Épaisseur du tissu palissadique, diamètre moyen du cylindre central et des canaux résinifères.

Mean thickness of palissadic tissue (tp), ratio : $\mathrm{E} / \mathrm{tp}$, mean diameter of the central cylinder and mean diameter of the resiniferous canals

\begin{tabular}{|c|c|c|c|c|c|c|c|c|}
\hline Espèce & Paramètre & C.A. & C.N. & C.S. & C.E. & C.C. & C.O. & P.D. \\
\hline \multirow{4}{*}{$\begin{array}{l}\text { Abies alba } \\
\text { Ecouves II }\end{array}$} & $\begin{array}{l}\text { Épaisseur }(\mu) \text { tissu palissa- } \\
\text { dique }\left(t_{p}\right)\end{array}$ & 94,8 & 117,9 & 134,5 & 131,7 & 134,9 & 122,5 & 141,3 \\
\hline & $\mathrm{E} / \mathrm{t}_{\mathrm{p}}$ & 5,1 & 4,6 & 4,4 & 4,0 & 4,2 & 4,1 & 4,2 \\
\hline & Diamètre cylindre central $(\mu)$ & 167,9 & 198,8 & 194,2 & 187,1 & 193,1 & 183,9 & 187,1 \\
\hline & $\begin{array}{l}\text { Diamètre canaux résinifères } \\
(\mu)\end{array}$ & 82,7 & 89,8 & 90,2 & 84,8 & 83,8 & 85,6 & 88,0 \\
\hline \multirow{4}{*}{$\begin{array}{l}\text { Abies alba } \\
\text { Canigou }\end{array}$} & $\begin{array}{l}\text { Épaisseur }(\mu) \text { tissu palissa- } \\
\text { dique }\left(\mathrm{t}_{\mathrm{p}}\right)\end{array}$ & 109,3 & 167,2 & 145,9 & 139,5 & 163,3 & 142,4 & 204,8 \\
\hline & $\mathrm{E} / \mathrm{t}_{\mathrm{p}}$ & 4,6 & 3,7 & 3,9 & 4,1 & 3,8 & 4,0 & 3,5 \\
\hline & Diamètre cylindre central $(\mu)$ & 175,0 & 200,2 & 188,9 & 192,8 & 205,9 & 194,9 & 186,0 \\
\hline & $\begin{array}{l}\text { Diamètre canaux résinifères } \\
(\mu)\end{array}$ & 74,2 & 94,1 & 90,2 & 90,5 & 90,9 & 95,9 & 87,0 \\
\hline \multirow{4}{*}{$\begin{array}{l}\text { Abies } \\
\text { nordmanniana }\end{array}$} & $\begin{array}{l}\text { Épaisseur }(\mu) \text { tissu palissa- } \\
\text { dique }\left(t_{p}\right)\end{array}$ & 89,5 & 159,4 & 171,1 & 156,6 & 202,4 & 138,1 & 160,5 \\
\hline & $\mathrm{E} / \mathrm{t}_{\mathrm{p}}$ & 5,1 & 3,7 & 3,9 & 3,8 & 3,3 & 4,0 & 3,9 \\
\hline & Diamètre cylindre central $(\mu)$ & 176,1 & 202,0 & 218,3 & 212,3 & 220,1 & 194,2 & 205,5 \\
\hline & $\begin{array}{l}\text { Diamètre canaux résinifères } \\
(\mu)\end{array}$ & 78,1 & 81,3 & 95,5 & 79,2 & 86,3 & 81,7 & 85,2 \\
\hline \multirow{4}{*}{$\begin{array}{l}\text { Pseudotsuga } \\
\text { menziesii }\end{array}$} & $\begin{array}{l}\text { Épaisseur }(\mu) \text { tissu palissa- } \\
\text { dique }\left(t_{p}\right)\end{array}$ & 82,4 & 126,7 & 111,8 & 115,4 & 99,4 & 96,6 & 145,2 \\
\hline & $\mathrm{E} / \mathrm{t}_{\mathrm{p}}$ & 4,3 & 3,7 & 4,2 & 3,7 & 4,3 & 4,6 & 3,5 \\
\hline & Diamètre cylindre central $(\mu)$ & 128,2 & 176,8 & 178,2 & 161,9 & 170,4 & 172,5 & 186,7 \\
\hline & $\begin{array}{l}\text { Diamètre canaux résinifères } \\
(\mu)\end{array}$ & 22,7 & 43,7 & 51,1 & 40,8 & 41,5 & 45,1 & 55,0 \\
\hline
\end{tabular}

les trois sapins et le douglas, les aiguilles de la coupe d'abri sont plates, minces et présentent des bordures très aiguës alors que dans le cas du plein découvert elles sont beaucoup plus épaisses. Le rapport 1/E qui rend compte de la forme de la section de l'aiguille est toujours le plus faible en plein découvert. Les aiguilles de la clairière sont intermédiaires entre celles de la coupe d'abri et celles du plein découvert. Ces résultats sont voisins de ceux trouvés par TRONCHET (1956) qui a montré que l'épaisseur des feuilles du chêne rouvre augmentait avec l'éclairement relatif. Les types intermédiaires seront difficiles à mettre en évidence. Chez l'épicéa le même effet existe mais il apparaît sur le rapport $\mathrm{D} / \mathrm{d}$, les aiguilles sont aplaties dans la coupe d'abri (D/d élevé) et « globuleuses » dans le plein découvert ( $D / d$ faible). 
Si l'on compare ces traitements à ce qui se passe dans un couvert forestier fermé, il est probable que l'on retrouvera ces trois grands types d'aiguilles variant du haut en bas des houppiers.

En ce qui concerne les stomates l'effet du traitement n'est pas toujours significatif alors que l'on aurait pu imaginer le contraire. En effet, certains auteurs (GINDEL, 1969) ont montré que le nombre et la dimension des stomates étaient sensibles aux conditions de milieu. Les traitements diffèrent essentiellement dans ces expériences par l'énergie reçue et par les conditions d'alimentation en eau. Il aurait donc fallu étudier l'effet de chacun de ces facteurs. Mais dans la nature il y a une interrelation entre ces paramètres. En effet à des éclairements importants correspondent des conditions d'alimentation en eau difficile (ETP élevé) pour les jeunes plants. Quand l'éclairement diminue les conditions d'alimentation en eau s'améliorent car l'évapotranspiration potentielle baisse au niveau des feuilles.

D'après les études effectuées par divers auteurs (Dufour, 1887, SCHOCH, 1972) le nombre de stomates augmenterait avec l'énergie lumineuse reçue et l'état de siccité du sol. Ainsi dans le cas présent le traitement de plein découvert devrait présenter le nombre de stomates le plus élevé. Les autres traitements devraient avoir dans leur ensemble, une numération stomatique inférieure. C'est vrai dans certains cas, Douglas et Abies alba (Ecouves) (figure 8) mais pas dans tous. Dans ce cas précis, il est donc difficile de tirer des numérations stomatiques une conclusion quant à la signification écologique des traitements.

Les dimensions des stomates selon certains auteurs (De PARCevaux, 1964) varient avec la sécheresse du sol et de l'air et seraient plus faibles dans les stations « sèches ». Compte tenu des conditions microécologiques de nos traitements, les stomates devraient être plus petits dans le plein découvert et la coupe d'abri. C'est ce qui se produit pour les trois sapins sans que l'effet soit significatif au seuil de probabilité choisi. De ce point de vue, les différences écologiques ne sont sans doute pas suffisantes pour entraîner des variations significatives.

En ce qui concerne les dimensions des aiguilles, il apparaît que les 2 provenances de sapin ont des dimensions très voisines et que sur ce plan-là, il semble que l'on ne puisse pas espérer les distinguer. GatTi (1970) qui a étudié plusieurs provenances de Pseudotsuga menziesii est arrivé aux mêmes conclusions.

Le nombre et les dimensions des stomates sont du même ordre de grandeur que ceux cités par certains auteurs (ARENA, 1960, NAPP, Zirn, 1966). Dans l'ensemble, remarquons que les deux provenances de Sapin pectiné et le Sapin de Nordmann ont des numérations stomatiques très voisines (60-80 stomates $/ \mathrm{mm}^{2}$ surface foliaire chez Abies alba Ecouves II, 62 à 78 st $/ \mathrm{mm}^{2}$ chez Abies alba Canigou, 63 à $86 \mathrm{st} / \mathrm{mm}^{2}$ chez Abies nordmanniana). Par contre, le douglas a une numération stomatique beaucoup plus élevée (de 110 à 142 stomates $/ \mathrm{mm}^{2}$ de feuille selon les traitements). Les dimensions des stomates des 2 provenances de sapin pectiné et de sapin de Nordmann sont aussi très voisines; par contre, elles sont beaucoup plus faibles chez le douglas. Il y a là incontestablement une différence importante entre les sapins et le douglas, qui a une densité stomatique plus élevée et des stomates plus petits. Il est possible que ces caractéristiques correspondent à une meilleure adaptation du Douglas vis-à-vis de l'économie de l'eau $\left({ }^{1}\right)$. En effet, il est actuellement prouvé (Stalfelt, 1932, De Parcevaux, 1964) que des stomates nombreux et petits permettent une meilleure régulation de la transpiration que des stomates peu nombreux et grands. De ce point de vue, il serait sans doute

(1) Cette conclusion est recoupée par des observations de terrain qui montrent que le sapin est plus sensible à la sécheresse que le douglas. 


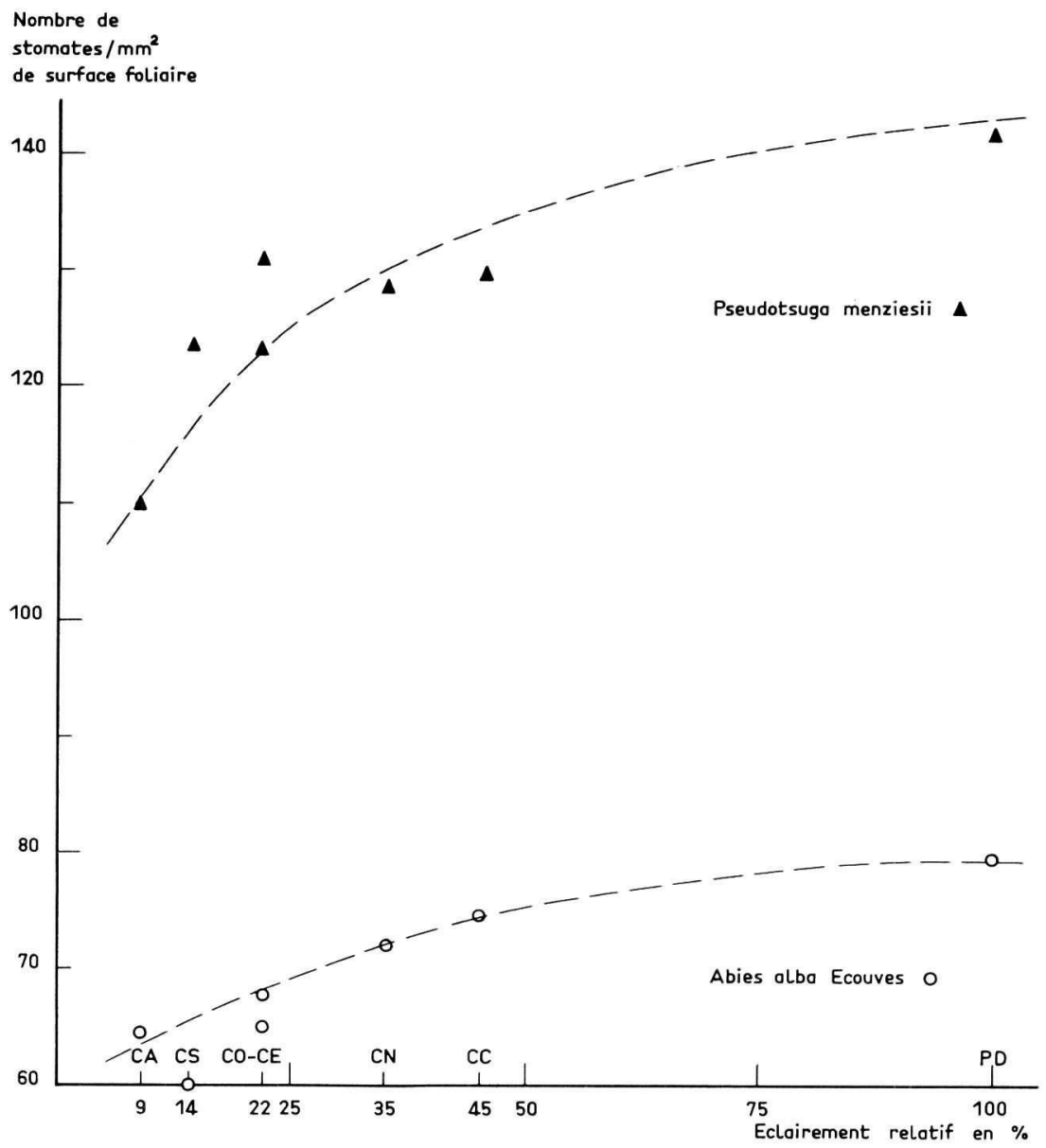

FIG. 8. - Variation du nombre de stomates par $\mathrm{mm}^{2}$ de surface foliaire en fonction de l'éclairement relatif

Variations of stomatas number (by $\mathrm{mm}^{2}$ ) in function of relative illumination

intéressant d'étudier pour différentes provenances de Douglas la variabilité de ces caractères : nombre et dimensions des stomates.

On peut penser que ce double caractère reflétant l'adaptation d'une espèce ou provenance doit être recherché chez les essences offrant une grande variabilité d'adaptation aux facteurs écologiques. Il semble en effet que pour 100 cellules épidermiques, le nombre de stomates soit génétiquement fixé. Ce qui varierait ce serait la taille des cellules selon l'importance de la sécheresse.

L'étude effectuée montre également que l'influence du traitement et, par voie de conséquence des facteurs microclimatiques responsables d'une croissance différente (AusSENAC, en préparation), ne se traduit pas au niveau anatomique et morphologique par une accommodation aussi fine que nous aurions pu l'espérer a priori. 
A partir des résultats obtenus ici, il est possible de comprendre la crise dite de découvert chez les plants ayant poussé à l'ombre et qui sont brusquement éclairés par enlèvement du couvert. Quand le niveau d'énergie ${ }^{1}$ ) reçu par les arbres est très bas (d'après ce que nous avons observé $<14 \%$ ), les aiguilles des résineux (sapin pectiné, sapin de Nordmann, Épicéa, Douglas) ont une morphologie et une anatomie très particulière, très facilement identifiable aussi bien en coupe microscopique qu'en examen macroscopique. Cette organisation est très différente (tissu palissadique très mince) de celle des aiguilles de pleine lumière. Quand le niveau d'éclairement augmente soudain, les aiguilles s'avèrent incapables d'utiliser l'énergie dispensée $\left({ }^{2}\right)$. BOYSEN JENSEN (1932) l'a déjà montré pour les feuilles de hêtre. L'arbre ne pourra bénéficier de l'éclairement supplémentaire disponible que quand de nouvelles aiguilles seront nées avec une structure et une physiologie adaptée.

Les plants résineux en clairière au contraire présentent des aiguilles dont la morphologie et la structure anatomique ne sont pas très différentes des aiguilles de plein découvert. Il paraît très probable, l'examen anatomique le laisse supposer, que ces deux types d'aiguilles ont des courbes d'assimilation voisines, ainsi après enlèvement du couvert latéral, les aiguilles des «plants de clairière » sont capables de bénéficier des nouvelles conditions d'éclairements. Ce type de traitement permet donc un passage sans problème de l'abri temporaire au plein découvert. En fin de compte, toute coupe d'abri que les forestiers appellent « sombre » est extrêmement dangereuse pour l'avenir d'une plantation, même s'il s'agit d'une essence d'ombre qui, au départ, semble s'en trouver bien.

Lorsque la coupe rase n'est pas supportable, le gestionnaire de forêt doit s'orienter vers une coupe d'abri aussi claire que possible dès la première année ou, mieux encore peut-être, vers des coupes par bandes qui sont en somme, de notre point de vue, des «clairières jointives $\gg$ !

\section{Reçu pour publication en juin 1973.}

\section{REMERCIEMENTS}

Je remercie ici : - M. Millier qui a effectué le traitement statistique des données, travail.

- Mesdemoiselles Bernage et Grand, Messieurs Desjeunes et Willm qui ont participé à ce

\section{SUMMARY}

EFFECTS OF SOME DIFFERENT MICROCLIMATIC CONDITIONS ON MORPHOLOGY AND ANATOMIC STRUCTURE OF NEEDLES

Five species have been studied : Abies alba (Ecouves II), Abies alba (Canigou), Abies nordmaniana (Gerbeviller), Picea abies (La Joux), Pseudotsuga menziesii (Farges II). Trees have been planted according to seven treatments :

— under a shelterwood felling ( $\mathrm{n} / \mathrm{ha}: 1300$ - mean heigth : $13 \mathrm{~m})$.

(1) Le niveau d'énergie est exprimé en pourcentage de l'éclairement incident (éclairement relatif).

(2) Il est possible que les aiguilles d'ombre aient en plein découvert une mauvaise régulation stomatique qui se traduirait par un déficit hydrique important et par voie de conséquence par une élévation de leur température (échauffement). 
— five situations (north, south, east, west, centre) in a circular clearing (diameter : $26 \mathrm{~m}$ - mean height of surrounding stand : $13 \mathrm{~m}$ ).

- in a cleared area.

Light and evaporation capacity of the air in those treatments are quite different.

Measurements were : mensuration and section of needles, number and size of stomatas.

There is a good relation between the form of the needles and the level of incident energy. In general, the needles from shelterwood felling are flat then they are more thicker in the cleared aera. The ration width/thickness decreases from little relative illumination (shelterwood aera) to largest eclairements. The same thing is observed for the ratio of diagonals of the section from needles of Picea abies. The ratio length/width changes with relative illumination and has a maximum in the clearing. Same thing for the lenght of needles.

For Douglas fir and Abies alba (Ecouves II) the number of stomatas raises with eclairements; so it is greater in cleared aera. Douglas fir has a stomatic numeration larger and stomatas smaller than fir. These caracteristics are perhaps an indication of a better regulation of the transpiration.

The results of this work contribute to understand the crisis by clear cutting for plants which have grown in shadow and which are suddenly lighted by elimination of the cover.

When the level of incident ernergy is very low (14\%) needles have a morphology and anatomy very different from those of the cleared aera. When energy level raises the needles are « unable » to use this ernergy. In the contrary the trees in the clearing have their needles similar enough to those of the cleared aera and are able to profit by a large eclairement. This treatment allows a problemless way from temporary shelter to cleared aera. So, when clear cutting is not endurable, the forester is to use a shelterwood as clear as possible or, better, a strip - cutting.

\section{ZUSAMMENFASSUNG}

\section{DER EINFLUSS VERSCHIEDENER MIKROKLIMATE AUF DIE MORPHOLOGIE}

UND DIE ANATOMISCHE STRUKTUR DER NADELN EINIGER KONIFEREN

Folgende Holzarten und Herkünfte wurden untersucht : Abies alba : Écouves II. Abies alba: Canigou. Abies Nordmanniana : Gerbéviller. Picea abies : La Joux. Pseudotsuga menziesii : Farges II.

Die Pflanzen wurden auf 7 mikroklimatisch verschiedenen Standorten eingebracht.

— Schirmschlag (Stammzahl : 1 300/ha, mittlere Höhe : 13 m).

- Lochhieb (Durchmesser : 26 m, mittlere Höhe : 13 m), wobei die 5 Positionen Nord, Sud, West, Ost und Mitte unterschieden wurden.

- Kahlfläche.

Diese 7 Behandlungen unterscheiden sich im wesentlichen durch ihr Lichtklima und das Verdunstungsvermögen der Luft.

Die Untersuchungen betrafen morphologische Messungen an Nadeln und Nadelquerschnitten, sowie Messung und Zählung der Stomata.

Es besteht eine enge Beziehung zwischen der Form der Nadeln und der erhaltenen Strahlungsenergie. Im allgemeinen sind die Nadeln der unter Schirm stehenden Pflanzen flachgedrückt, während sie auf der Freifläche wesentlich dicker sind.

Das Verhältnis von Nadelbreite zu Nadeldicke nimmt mit zunehmender relativer Beleuchtung ab. Dies ist auch bei den Durchmessern der Nadelpaare der Fichte festzustellen.

Das Verhältnis von Nadellänge zu Nadelbreite verändert sich gleichfalls bei verschiedener relativer Beleuchtung und zeigt ein Maximum im Lochhieb. Dies lässt sich auch bei der Nadellänge feststellen.

Bei der Douglasie und der Tanne (Ecouves II) nimmt die Anzahl der Stomata mit steigender Beleuchtung zu und ist daher auf der Freifläche am höchsten. Die Douglasie hat eine grössere Anzahl Spaltöffnungen als die Tanne, jedoch sind die Stomata kleiner. Dies könnte auf ein besseres Regulationsvermögen bei der Transpiration hinweisen. 
Die Ergebnisse dieser Untersuchung erlauben die oft beobachtete Wachstumskrise bei plötzlicher Freistellung zu erklären.

Pflanzen die bei sehr geringem Lichtgenuss $(14 \%)$ aufgewaschen sind haben vis à vis dem Freistandpflanzen eine sehr verschiedene Morphologie und Anatomie der Nadeln. Erhöht sich plötzlich der Lichtgenuss, so sind diese Pflanzen nicht in der Lage die erhöhte Lichtmenge entsprechend auszunützen.

Die Pflanzen des Lochhiebes haben jedoch annähernd den gleichen Nadelaufbau als die Freistandpflanzen und können daher auf einen erhöhten Lichtgenuss positiv reagieren. Der Lochhieb ermöglicht daher nach einer zeitlichen Schutzwirkung in den ersten Jahren eine darauffolgende Freistellung ohne Schwierigkeiten. In Fällen wo die Pflanzung im Freistand nicht durchführbar erscheint, empfiehlt sich dem Waldbauer der Lochhieb oder noch besser ein streifenweiser Kahlschlag.

\section{RÉFÉRENCES BIBLIOGRAPHIQUES}

Aussenac G., 1972. - Climat, microclimat et production ligneuse. Ann. Sci.forest., (à paraître).

Arena M., 1960. - Anatomia comparativa di alcumi Organi vegetative de Abies nebrodensis (Lojac.) Mattei e Abies alba Mill. Atti Acad. naz. Lincei. Rc, Ser. VIII. Cl. Sci. fis. matemat., 29, 586-596.

Boysen-Jensen P., 1932. - Die Stoffproduktion der Pflanzen. Gustav Fischer, Verlagsbuchhandlung, Jena, Germany. Daguillon A., 1890. - Recherches morphologiques sur les feuilles de cônifères. Thèse Paris, Klincksieck,
85.

Dufour L., 1887. - Influence de la lumière sur la forme et la structure des feuilles. Thèse Paris, Masson, 413.

Gattı F., 1970. - Il riconoscimento di alcune provenienze di Pseudotsuga menziesii (Mirb.) Franco en base alle caratteristiche anatomiche degli aghi. Ann. Inst. sper. Silvicolt., 1, 327-341.

Gindel I., 1969. - Stomatal number and size as related to soil moisture in tree xerophytes in Israel. Ecology, 50, 2, 263-267.

JACKSON L. W. R., 1967. - Effect of shade on leaf structure of deciduous tree species. Ecology, 498-499.

Mer M. E., 1883. - De l'influence de l'ombre et de la lumière sur la structure et la végétation des aiguilles d'Abies excelsa. Bull. Soc. bot. Fr., 30, 2, 40-50.

Parcevaux (DE), 1963. - Transpiration végétale et production de matière sèche. Essai d'interprétation en fonction des facteurs du milieu. Ann. agron., 14 (5), 655-742.

Perrin, 1963. - Sylviculture. T. 1 : Bases scientifiques de la sylviculture. E.N.E.F., Nancy, 318.

Roussel L., 1962. - Étude théorique élémentaire de la trouée et de la bande. Soc. forest. Franche-Comté, 2 , $32,552-560$.

Roussel L., 1970. - La notion du niveau d'énergie et son utilisation en sylviculture. R.F.F., 2, 131-138.

Roussel L., 1972. — Photologie forestière. 144, Masson, Paris.

Sсносн P. G., 1972. - Variation de la densité stomatique de Capsicum annum L. en fonction du rayonnement global. C.R. Acad. Sc. Paris, 274, 2496-2498.

Tronchet A., Grangirard A., 1956. - L'analyse histométrique et son application à l'écologie forestière.

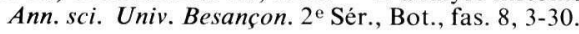

